\title{
Bergan-Wang Solution of Clamped Moderately Thick Rectangular Plates
}

\author{
Kamal Hassan ${ }^{1}$, Samer Guirguis ${ }^{2}$, Hassan El-Hamouly ${ }^{2}$ \\ 1. The British University in Egypt Cairo, Egypt. 2. Military Technical College, Cairo, Egypt.
}

Received: May 13, 2017 / Accepted: June 11, 2017 / Published: June 25, 2017

\begin{abstract}
Bergan-Wang approach has one governing equation in one variable only, namely the transverse deflection of a moderately thick plate. This approach faces no numerical difficulties as the thickness becomes very small. The solution of a fully clamped rectangular plate is presented using two different series solutions. The results of a square plate are compared with the results of the classical plate theory, Reissner- Mindlin theory and the three dimensional theory of elasticity for different aspect ratios. Two types of clamped boundary conditions are investigated. The obtained results show that Bergan-Wang approach gives good agreement for both very thin and moderately thick plates.
\end{abstract}

Keywords: Moderately thick plate, eighth-order partial differential equation, Reissner-Mindlin theory, Bergan-Wang approach, clamped boundary conditions.

\section{Introduction}

Plates might be classified according to thickness $(h)$ to $\operatorname{span}(a)$ ratio as follows: very thin plate $\frac{h}{a}<\frac{1}{100}$, moderately thin plate $\frac{1}{100}<\frac{h}{a}<\frac{1}{20}$, moderately thick $\frac{1}{20}<\frac{h}{a}<\frac{1}{8}$, for thick plate $\frac{1}{8}<\frac{h}{a}<\frac{1}{4}$ and very thick plates $\frac{\mathrm{h}}{\mathrm{a}}>\frac{1}{4}$ three-dimensional theory of elasticity should be applied. Classical plate theory [1-3] is widely accepted for the analysis of very thin plates. The governing equation of classical plate theory is:

$$
\Delta^{2} w=\frac{P}{D}
$$

where $\mathrm{w}$ is the transverse deflection of the plate mid-plane, $\mathrm{D}=\frac{\mathrm{Eh}^{3}}{12\left(1-v^{2}\right)}$ is the flexural rigidity, $\mathrm{E}$ is

Corresponding author: Kamal Hassan, Professor of mathematical engineering, The British University in Egypt,Faculty of Engineering,Basic Sciences Department, E-mail: kamal.hassan@bue.edu.eg.
Young's modulus, $v$ is the Poisson's ratio, $\Delta \equiv \frac{\partial^{2}}{\partial \mathrm{x}^{2}}+\frac{\partial^{2}}{\partial \mathrm{y}^{2}}$ is the Laplacian operator and $\mathrm{P}$ is the applied distributed transverse load.

The inclusion of shear deformation in plate deflection [4] is recommended in general in case of moderately thin and moderately thick plates. It is also recommended for very thin plates in locations of bending-stress concentrations such as near sudden changes in thickness or support conditions and near holes whose dimensions are comparable with the thickness or re-entered corners, and of course when studying composite materials.

Reissner-Mindlin theory [5-7] is the plate theory that is most used in engineering applications. This theory leads to a sixth-order system of governing partial differential equations in three unknowns, namely, the transverse deflection of the middle plane $\mathrm{w}$ and the two rotations of the normal to the middle plane $\theta_{\mathrm{x}}$ and $\theta_{\mathrm{y}}$ (Fig.1).

Due to the numerical difficulties encountered with Reissner-Mindlin theory in case of very thin plates [8], Bergan and Wang [9-10] 


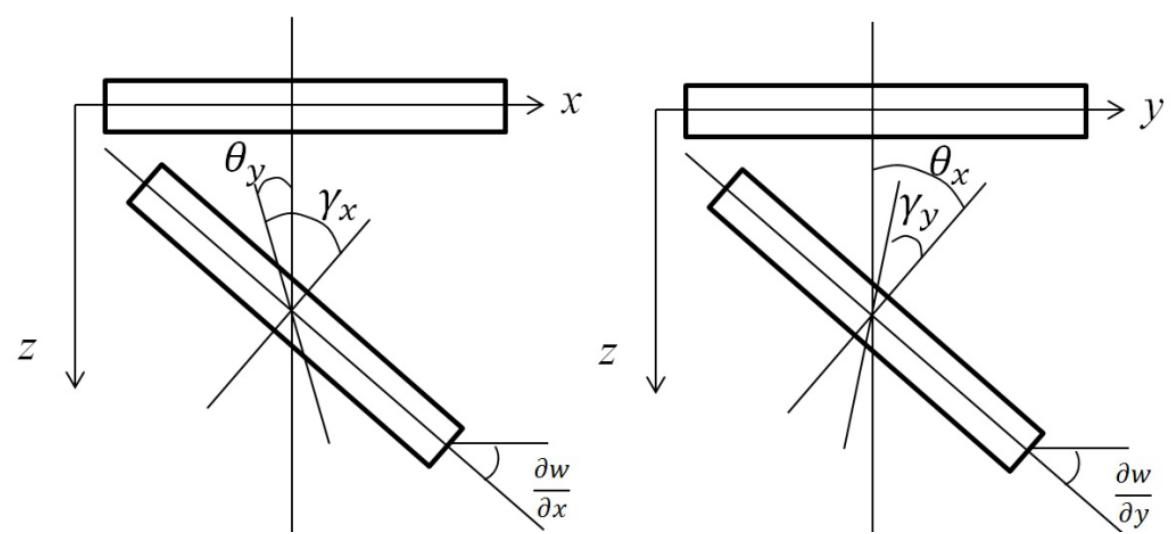

Fig. 1 Deflection of a thick plate $\left(\gamma_{x}\right.$ and $\gamma_{y}$ are the rotations of the normal due to shear only while $\theta_{x}$ and $\theta_{y}$ are the total rotations due to both bending and shear).

proposed an approach which led to one partial differential equation of the eighth order of the transverse deflection $\mathrm{w}$. This approach faces no difficulties as $\frac{h}{a}$ becomes very small. According to this approach, the strain energy per unit area, $F$ can be written as the sum of bending contribution $F_{B}$ and shearing contribution $F_{S}$. so $F=F_{B}+F_{S}$.

Where $F_{B}=\frac{1}{2} K_{B}^{T} D_{B} K_{B} \quad$ and $\quad F_{S}=\frac{1}{2} K_{S}^{T} D_{S} K_{S}$ in which ()$^{T}$ denotes the transpose of a matrix and the suffix $B(S)$ stands for bending (shear).

For a homogeneous and isotropic material, the above matrices are given by:

$$
\begin{gathered}
K_{B}=\left[\begin{array}{c}
w_{, x x}-\gamma_{x, x} \\
w_{, y y}-\gamma_{y, y} \\
2 w_{, x y}-\gamma_{x, y}-\gamma_{y, x}
\end{array}\right] \\
K_{S}=\left[\begin{array}{l}
\gamma_{x} \\
\gamma_{y}
\end{array}\right]
\end{gathered}
$$

With

$$
\begin{gathered}
\gamma_{x}=-h_{0}^{2}\left(w_{, x x x}+w_{, y y x}\right) \\
\gamma_{y}=-h_{0}^{2}\left(w_{, y y y}+w_{, x x y}\right) \\
D_{B}=\frac{E h^{3}}{12\left(1-v^{2}\right)}\left[\begin{array}{llc}
1 & v & 0 \\
v & 1 & 0 \\
0 & 0 & \frac{1-v}{2}
\end{array}\right] \\
D_{S}=\frac{5 E}{12(1+v)}\left[\begin{array}{ll}
1 & 0 \\
0 & 1
\end{array}\right] \\
h_{0}^{2}=\frac{h^{2}}{5(1-v)}
\end{gathered}
$$

The moments (Fig.2) are given by:

$$
\begin{gathered}
M=\left[\begin{array}{l}
M_{x x} \\
M_{y y} \\
M_{x y}
\end{array}\right] \\
=D_{B}\left[\begin{array}{c}
w_{, x x}+h_{0}^{2}\left(w_{, x x x x}+w_{, y y x x}\right) \\
w_{, y y}+h_{0}^{2}\left(w_{, y y y y}+w_{, x x y y}\right) \\
2\left(w_{, x y}+h_{0}^{2}\left(w_{, x x x y}+w_{, y y y x}\right)\right)
\end{array}\right]
\end{gathered}
$$

The shear forces are given by:

$$
Q=D_{S}\left[\begin{array}{l}
w_{, x x x}+w_{, y y x} \\
w_{, y y y}+w_{, x x y}
\end{array}\right] .
$$

The governing equation for Bergan-Wang approach is:

$$
\Delta^{2} w+h_{0}^{2} \Delta^{3} w+h_{0}^{4} \Delta^{4} w=\frac{P}{D}
$$

This equation clearly converges to the classical fourth order partial differential equation as $\mathrm{h}_{0}^{2}$ becomes very small. The difficulties of Bergan-Wang approach are the high order of the partial differential equation and the increased number of boundary conditions. A closed form solution for the case of simply supported square plate is given in [10] for different loadings. The clamped boundary conditions are more difficult [2].

In this work, two different series solutions of clamped rectangular plate (Fig. (2)) are used, namely:

$$
\begin{gathered}
w(x, y)=\sum_{m=1}^{\infty} \sum_{n=1}^{\infty} W_{m n} \\
\left(1-\cos \left(2 \alpha_{m} x\right)\right)\left(1-\cos \left(2 \beta_{n} y\right)\right),
\end{gathered}
$$




$$
w(x, y)=\frac{4}{a b} \sum_{m=1}^{\infty} \sum_{n=1}^{\infty} W_{m n}^{*} \sin \left(\alpha_{m} x\right) \sin \left(\beta_{n} y\right)
$$

where $\alpha_{m}=\frac{\pi m}{a}, \beta_{n}=\frac{\pi n}{b}$ and $a, b$ are the side dimensions of the rectangular plate (Fig.2). For the case of classical plate theory, similar series are proposed $[11,12]$.This is the first time, to the best knowledge of the authors, that such solutions were used for clamped plates based on Bergan-Wang approach.

The coefficients: $W_{m n}$ and $W^{*}{ }_{m n}$ are to be determined according to the method of solution. Because these two series are used to get the classical plate theory solutions, we can directly get separate expressions of the solutions due to bending and due to shear. This is in harmony with Shimpi's work $[13,14]$ and Reddy's solutions [7, 3].

The methods of solution are given for a clamped rectangular plate. The numerical results of a square plate are compared with the solution of classical plate theory, Reissner-Mindlin theory and the three dimensional theory of elasticity. For the moderately thick plates, the results are comparable. It is hoped that these results will serve as bench marks for finite element solution for Bergan-Wang approach.

\section{Boundary Conditions for a Clamped Plate}

The eighth-order differential equation needs four boundary conditions on each side of the plate. This is a problem in itself, because the boundary conditions of Reissner-Mindlin's theory are only three which goes with the sixth-order of the system of equations governing the theory. Moreover, it is intuitively "reasonable" to have only three boundary conditions.

A comparison with the case of classical plate theory, where the clamped boundary conditions are the imposition of zero values for $\mathrm{w}$ and its normal derivative $\frac{\partial \mathrm{w}}{\partial \mathrm{n}}$, one is motivated to impose, along each clamped edge, zero values for $\mathrm{w}$ and its normal first, second and third derivatives ( $\mathrm{n}$ can be $\mathrm{x}$ or $\mathrm{y}$ according to which direction is normal to the edge under study). This choice contradicts the mechanics of supports (the annulation of second normal derivative of $\mathrm{w}$ is a characteristic of a simple support). As a matter of fact, such a choice is a very severe clamping which cannot be realized in practice. Therefore, the boundary conditions were proposed to be; zero values for $\mathrm{w}, \frac{\partial \mathrm{w}}{\partial \mathrm{n}}, \frac{\partial^{3} \mathrm{w}}{\partial \mathrm{n}^{3}}$ and $\frac{\partial^{5} \mathrm{w}}{\partial \mathrm{n}^{5}}[10]$. Such a choice has fortunately been fulfilled by the series given in equation (3)

$$
\begin{gathered}
\sum_{m=1}^{\infty} \sum_{n=1}^{\infty} W_{m n}\left(1-\cos \left(2 \alpha_{m} x\right)\right) \times \\
\left(1-\cos \left(2 \beta_{n} y\right)\right) .
\end{gathered}
$$

According to Reissner-Mindlin, there are two types of clamped boundary conditions: The soft one, for which we impose zero values for $w, \theta_{n}$ and $M_{n s}$ (Fig.3) along each edge, and the hard one, where we impose zero values for $w, \theta_{n}$ and $\theta_{s}$ ( $s$ stands for tangential direction of the edge while $n$ stands for the normal to the edge under study) $[15,16]$.

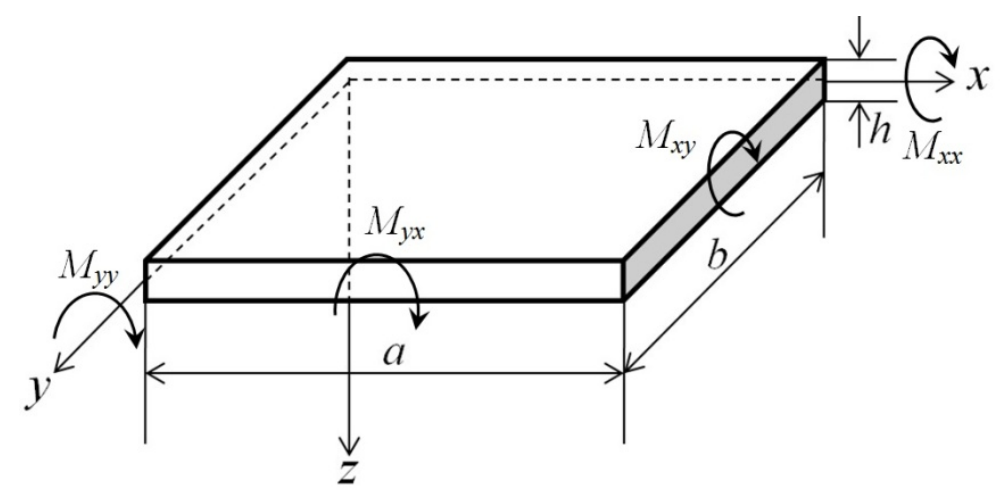

Fig. 2 The rectangular plate. 


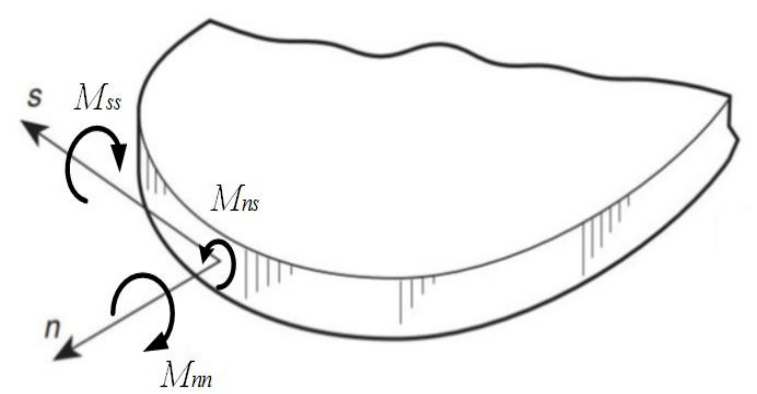

Fig. 3 Moments expressed in tangential and normal axes.

In [9], It has been shown that the expressions for $\theta_{n}, \theta_{s}$ and $M_{n s}$ are related to $w$ as follows:

$$
\begin{gathered}
\theta_{s}=\frac{\partial w}{\partial n}+h_{0}^{2}\left(\frac{\partial^{3} w}{\partial n^{3}}+\frac{\partial^{3} w}{\partial s^{2} \partial n}\right), \\
\theta_{n}=\frac{\partial w}{\partial s}+h_{0}^{2}\left(\frac{\partial^{3} w}{\partial s^{3}}+\frac{\partial^{3} w}{\partial n^{2} \partial s}\right), \\
M_{n s}=2\left(w_{, n s}+h_{0}^{2}\left(w_{, n n n s}+w_{, s s s n}\right)\right)
\end{gathered}
$$

In this work, the soft clamped case has been chosen in addition to the imposition of zero value to the normal derivative of $w$. This choice which will be denoted by BC-I goes well with the choice of the aforementioned series. Application of the well-known Galerkin method $[17,18]$ has been used to find out the coefficients $\mathrm{W}_{\mathrm{mn}}$ leading to very good results.

Another interesting type of boundary conditions has been investigated. In addition to the two main boundary conditions on $\mathrm{w}$ and $\frac{\partial \mathrm{w}}{\partial \mathrm{n}}$ used in the classical plate theory, two more ones come from the decomposition of the eighth-order equation into two fourth-order equations. In fact, we can write down the Bergan-Wang equation (2) as follows:

$$
\Delta^{2}\left(w+h_{0}^{2} \Delta w+h_{0}^{4} \Delta^{2} w\right)=\frac{P}{D}
$$

Assuming

$$
u=w+h_{0}^{2} \Delta w+h_{0}^{4} \Delta^{2} w,
$$

Then

$$
\Delta^{2} u=\frac{P}{D}
$$

If we solve the first equation for $\mathrm{u}$ with boundary conditions: $\mathrm{u}=0$ and $\frac{\partial \mathrm{u}}{\mathrm{n}}=0$ then we solve the second equation for $\mathrm{w}$ with the two boundary conditions: $\mathrm{w}=0$ and $\frac{\partial \mathrm{w}}{\partial \mathrm{n}}=0$. Therefore, this type of boundary conditions, which will be denoted by $\mathrm{BC}-\mathrm{II}$, is the imposition of zero values along the clamped edge for $\mathrm{w}, \frac{\partial \mathrm{w}}{\partial \mathrm{n}},\left(\mathrm{w}+\mathrm{h}_{0}^{2} \Delta \mathrm{w}+\mathrm{h}_{0}^{4} \Delta^{2} \mathrm{w}\right)$ and

$$
\frac{\partial}{\partial \mathrm{n}}\left(\mathrm{w}+\mathrm{h}_{0}^{2} \Delta \mathrm{w}+\mathrm{h}_{0}^{4} \Delta^{2} \mathrm{w}\right)
$$

Clearly BC-II coincide well with the boundary conditions of the classical plate theory. Solving the problem needs only the usual methods of solving the classical plate theory with minor modifications.

An application of the finite integral transform [12, 19 and 20] has led to good results. Nevertheless, it is still believed that further investigations of the boundary conditions are needed.

\subsection{First Type of Clamped Boundary Conditions (Solved by GALERKIN'S Method)}

Consider a fully clamped isotropic rectangular plate of length $\mathrm{a}$ and width $\mathrm{b}$, where $0 \leq \mathrm{x} \leq \mathrm{a}$ and $0 \leq \mathrm{y} \leq \mathrm{b}$. The partial differential equation (2) governs the plate deflection. Applying the Laplacian operator in equation (2), gives the partial differential equation of the plate transverse deflection:

$$
\begin{gathered}
\frac{\partial^{4} w}{\partial x^{4}}+2 \frac{\partial^{4} w}{\partial x^{2} \partial y^{2}}+\frac{\partial^{4} w}{\partial y^{4}}+ \\
h_{0}^{2}\left(\frac{\partial^{6} w}{\partial x^{6}}+3 \frac{\partial^{6} w}{\partial x^{4} \partial y^{2}}+3 \frac{\partial^{6} w}{\partial x^{2} \partial y^{4}}+\frac{\partial^{6} w}{\partial y^{6}}\right)+ \\
h_{0}^{4}\left(\frac{\partial^{8} w}{\partial x^{8}}+4 \frac{\partial^{8} w}{\partial x^{6} \partial y^{2}}+6 \frac{\partial^{8} w}{\partial x^{4} \partial y^{4}}\right. \\
\left.+4 \frac{\partial^{8} w}{\partial x^{2} \partial y^{6}}+\frac{\partial^{8} w}{\partial y^{8}}\right)=\frac{P}{D}
\end{gathered}
$$

Assuming the solution to be of the form given in equation (3):

$$
w(x, y)=\sum_{m=1}^{\infty} \sum_{n=1}^{\infty} W_{m n}
$$




$$
\left(1-\cos \left(2 \alpha_{m} x\right)\right)\left(1-\cos \left(2 \beta_{n} y\right)\right) \text {. }
$$

Differentiate this proposed solution all the differentials that are needed to substitute in equation (2).

$$
\begin{gathered}
\sum_{m=1}^{\infty} \sum_{n=1}^{\infty} W_{m n} \times \\
{\left[\left\{-\left(2 \alpha_{m}\right)^{4} \cos \left(2 \alpha_{m} x\right)\left(1-\cos \left(2 \beta_{n} y\right)\right)+\right.\right.} \\
2\left(2 \alpha_{m}\right)^{2}\left(2 \beta_{n}\right)^{2} \cos \left(2 \alpha_{m} x\right) \cos \left(2 \beta_{n} y\right)- \\
\left.\left(2 \beta_{n}\right)^{4} \cos \left(2 \beta_{n} y\right)\left(1-\cos \left(2 \alpha_{m} x\right)\right)\right\}+ \\
h_{0}^{2}\left\{\left(2 \alpha_{m}\right)^{6} \cos \left(2 \alpha_{m} x\right)\left(1-\cos \left(2 \beta_{n} y\right)\right)-\right. \\
3\left(2 \alpha_{m}\right)^{4}\left(2 \beta_{n}\right)^{2} \cos \left(2 \alpha_{m} x\right) \cos \left(2 \beta_{n} y\right)- \\
3\left(2 \alpha_{m}\right)^{2}\left(2 \beta_{n}\right)^{4} \cos \left(2 \alpha_{m} x\right) \cos \left(2 \beta_{n} y\right)+ \\
\left.\left(2 \beta_{n}\right)^{6} \cos \left(2 \beta_{n} y\right)\left(1-\cos \left(2 \alpha_{m} x\right)\right)\right\}+ \\
h_{0}^{4}\left\{-\left(2 \alpha_{m}\right)^{8} \cos \left(2 \alpha_{m} x\right)\left(1-\cos \left(2 \beta_{n} y\right)\right)+\right. \\
4\left(2 \alpha_{m}\right)^{6}\left(2 \beta_{n}\right)^{2} \cos \left(2 \alpha_{m} x\right) \cos \left(2 \beta_{n} y\right)- \\
6\left(2 \alpha_{m}\right)^{4}\left(2 \beta_{n}\right)^{4} \cos \left(2 \alpha_{m} x\right) \cos \left(2 \beta_{n} y\right)+ \\
4\left(2 \alpha_{m}\right)^{2}\left(2 \beta_{n}\right)^{6} \cos \left(2 \alpha_{m} x\right) \cos \left(2 \beta_{n} y\right)- \\
\left.\left.\left(2 \beta_{n}\right)^{8} \cos \left(2 \beta_{n} y\right)\left(1-\cos \left(2 \alpha_{m} x\right)\right)\right\}\right]=\frac{P}{D}
\end{gathered}
$$

Multiply both side by

$$
\left(1-\cos \left(2 \alpha_{k} x\right)\right)\left(1-\cos \left(2 \beta_{1} y\right)\right)
$$

Where $k$ and $l$ can take any integer value from 1 to $\infty$.

Double integrate both sides of the equation with respect to $\mathrm{x}$ and $\mathrm{y}$ with the limits of integration are set to be $0 \rightarrow \mathrm{a}$ and $0 \rightarrow \mathrm{b}$ respectively.

$$
\begin{gathered}
R H S=\int_{0}^{a} \int_{0}^{b} \frac{P}{D}\left(1-\cos \left(2 \alpha_{k} x\right)\right) \times \\
\left(1-\cos \left(2 \beta_{l} y\right)\right) d x d y .
\end{gathered}
$$

For a homogeneous plate with uniform load:

$$
\begin{gathered}
R H S=\frac{P}{D} \int_{0}^{a} \int_{0}^{b}\left(1-\cos \left(2 \alpha_{k} x\right)\right) \times \\
\left(1-\cos \left(2 \beta_{l} y\right)\right) d x d y \\
=\frac{P}{D} \int_{0}^{a} \int_{0}^{b}\left\{\begin{array}{c}
1+\cos \left(2 \alpha_{k} x\right) \cos \left(2 \beta_{l} y\right)- \\
\cos \left(2 \alpha_{k} x\right)-\cos \left(2 \beta_{l} y\right)
\end{array}\right\} d x d y \\
=\frac{P}{D} a b .
\end{gathered}
$$

To find $L H S$ we start by performing the following integrals:

$$
\begin{aligned}
& \int_{0}^{a} \cos \left(2 \alpha_{m} x\right) \cos \left(2 \alpha_{k} x\right) d x\left\{\begin{array}{l}
\frac{a}{2} \text { if } m=k \\
0 \text { if } m \neq k
\end{array},\right. \\
& \int_{0}^{a} \cos \left(2 \alpha_{m} x\right) d x=0, \\
& \sum_{m=1}^{\infty} \sum_{n=1}^{\infty} \int_{0}^{a} \int_{0}^{b} W_{m n}\left[\left\{-\left(2 \alpha_{m}\right)^{4} \cos \left(2 \alpha_{m} x\right) \times\right.\right. \\
& \left(1-\cos \left(2 \beta_{n} y\right)\right)+ \\
& 2\left(2 \alpha_{m}\right)^{2}\left(2 \beta_{n}\right)^{2} \cos \left(2 \alpha_{m} x\right) \cos \left(2 \beta_{n} y\right)- \\
& \left.\left(2 \beta_{n}\right)^{4} \cos \left(2 \beta_{n} y\right)\left(1-\cos \left(2 \alpha_{m} x\right)\right)\right\}+ \\
& h_{0}^{2}\left\{\left(2 \alpha_{m}\right)^{6} \cos \left(2 \alpha_{m} x\right)\left(1-\cos \left(2 \beta_{n} y\right)\right)-\right. \\
& 3\left(2 \alpha_{m}\right)^{4}\left(2 \beta_{n}\right)^{2} \cos \left(2 \alpha_{m} x\right) \cos \left(2 \beta_{n} y\right)- \\
& 3\left(2 \alpha_{m}\right)^{2}\left(2 \beta_{n}\right)^{4} \cos \left(2 \alpha_{m} x\right) \cos \left(2 \beta_{n} y\right)+ \\
& \left.\left(2 \beta_{n}\right)^{6} \cos \left(2 \beta_{n} y\right)\left(1-\cos \left(2 \alpha_{m} x\right)\right)\right\}+ \\
& h_{0}^{4}\left\{-\left(2 \alpha_{m}\right)^{8} \cos \left(2 \alpha_{m} x\right)\left(1-\cos \left(2 \beta_{n} y\right)\right)+\right. \\
& 4\left(2 \alpha_{m}\right)^{6}\left(2 \beta_{n}\right)^{2} \cos \left(2 \alpha_{m} x\right) \cos \left(2 \beta_{n} y\right)- \\
& 6\left(2 \alpha_{m}\right)^{4}\left(2 \beta_{n}\right)^{4} \cos \left(2 \alpha_{m} x\right) \cos \left(2 \beta_{n} y\right)+ \\
& 4\left(2 \alpha_{m}\right)^{2}\left(2 \beta_{n}\right)^{6} \cos \left(2 \alpha_{m} x\right) \cos \left(2 \beta_{n} y\right)- \\
& \left.\left.\left(2 \beta_{n}\right)^{8} \cos \left(2 \beta_{n} y\right)\left(1-\cos \left(2 \alpha_{m} x\right)\right)\right\}\right] \times \\
& {\left[\left(1-\cos \left(2 \alpha_{k} x\right)\right)\left(1-\cos \left(2 \beta_{l} y\right)\right)\right] d x d y=} \\
& {\left[-\left(2 \alpha_{k}\right)^{4}+h_{0}^{2}\left(2 \alpha_{k}\right)^{6}-h_{0}^{4}\left(2 \alpha_{k}\right)^{8}\right] \times} \\
& {\left[-\frac{1}{2}\left[W_{k 1}+W_{k 2}+\ldots+W_{k(l-1)}+W_{k(l+1)}+\ldots\right]\right]+} \\
& {\left[-\left(2 \beta_{l}\right)^{4}+h_{0}^{2}\left(2 \beta_{l}\right)^{6}-h_{0}^{4}\left(2 \beta_{l}\right)^{8}\right] \times} \\
& {\left[-\frac{1}{2}\left[W_{1 l}+W_{2 l}+. .+W_{(k-1) l}+W_{(k+1) l}+. .\right]\right]+} \\
& \left\{\frac{3}{4}\left[\begin{array}{c}
\left(2 \alpha_{k}\right)^{4}-h_{0}^{2}\left(2 \alpha_{k}\right)^{6}+h_{0}^{4}\left(2 \alpha_{k}\right)^{8}+ \\
\left(2 \alpha_{k}\right)^{4}-h_{0}^{2}\left(2 \alpha_{k}\right)^{6}+h_{0}^{4}\left(2 \alpha_{k}\right)^{8}
\end{array}\right]+\right. \\
& 2\left(\frac{1}{4}\left(2 \alpha_{k}\right)^{2}\left(2 \beta_{l}\right)^{2}\right)+3 h_{0}^{2} \times \\
& \left(-\frac{1}{4}\left(2 \alpha_{k}\right)^{4}\left(2 \beta_{l}\right)^{2}-\frac{1}{4}\left(2 \alpha_{k}\right)^{2}\left(2 \beta_{l}\right)^{4}\right)+ \\
& 4 h_{0}^{4}\left(\frac{1}{4}\left(2 \alpha_{k}\right)^{6}\left(2 \beta_{l}\right)^{2}+\frac{1}{4}\left(2 \alpha_{k}\right)^{2}\left(2 \beta_{l}\right)^{6}\right) \\
& \left.+6 h_{0}^{4}\left(\frac{1}{4}\left(2 \alpha_{k}\right)^{4}\left(2 \beta_{l}\right)^{4}\right)\right\} W_{k l} \text {. }
\end{aligned}
$$


For $k=1 \rightarrow \mathrm{M}$ and $l=1 \rightarrow \mathrm{N}$ we perform the above integral $\mathrm{M} \times \mathrm{N}$ times to get $\mathrm{M} \times \mathrm{N}$ equations to be solved for the $\mathrm{M} \times \mathrm{N}$ unknowns $W_{k l}$.

After calculating the unknowns we substitute with their values in equation (11) to find the deflection at any point on the plate.

\subsection{Second Type of Clamped Boundary Conditions (Solved by Finite Integral Transform (FIT) Method}

This method involves solving the eighth degree partial differential equation of the plate transverse by solving two forth order differential equations consecutively. This is done as described in [12]. We solve equation (9) assuming the solution to be of the form:

$$
u(x, y)=\frac{4}{a b} \sum_{m=1}^{\infty} \sum_{n=1}^{\infty} U_{m n} \sin \left(\alpha_{m} x\right) \sin \left(\beta_{n} y\right),
$$

where

$$
U_{m n}=\int_{0}^{a} \int_{0}^{b} u(x, y) \sin \left(\alpha_{m} x\right) \sin \left(\beta_{n} y\right) .
$$

The boundary conditions on $\mathrm{u}$ are applied by imposing zero values to

$$
\left.u\right|_{x=0},\left.\quad u\right|_{y=0},\left.u\right|_{x=a},\left.\quad u\right|_{y=b},\left.\frac{\partial u}{\partial x}\right|_{x=0},\left.\frac{\partial u}{\partial y}\right|_{y=0},
$$

$\left.\frac{\partial \mathrm{u}}{\partial \mathrm{x}}\right|_{\mathrm{x}=\mathrm{a}}$, and $\left.\frac{\partial \mathrm{u}}{\partial \mathrm{y}}\right|_{\mathrm{y}=\mathrm{b}}$.

After solving (19), the solution is substituted in equation (18) to obtain the following partial differential equation:

$$
\begin{aligned}
w+h_{0}^{2}\left(\frac{\partial^{2} w}{\partial x^{2}}+\right. & \left.\frac{\partial^{2} w}{\partial y^{2}}\right) \\
& +h_{0}^{4}\left(\frac{\partial^{4} w}{\partial x^{4}}+2 \frac{\partial^{4} w}{\partial x^{2} \partial y^{2}}\right. \\
& \left.+\frac{\partial^{4} w}{\partial y^{4}}\right)=u(x, y)
\end{aligned}
$$

Again a double finite sine integral transform is used.

$$
w(x, y)=\frac{4}{a b} \sum_{m=1}^{\infty} \sum_{n=1}^{\infty} W_{m n} \sin \left(\alpha_{m} x\right) \sin \left(\beta_{n} y\right)
$$

$$
W_{m n}=\int_{0}^{a} \int_{0}^{b} w(x, y) \sin \left(\alpha_{m} x\right) \sin \left(\beta_{n} y\right)
$$

The same procedure is repeated where the boundary conditions are applied by imposing zero values to $\left.w\right|_{x=0},\left.w\right|_{y=0},\left.w\right|_{x=a},\left.w\right|_{y=b}$,

$$
\left.\frac{\partial w}{\partial x}\right|_{x=0},\left.\frac{\partial w}{\partial y}\right|_{y=0},\left.\frac{\partial w}{\partial x}\right|_{x=a}, \text { and }\left.\frac{\partial w}{\partial y}\right|_{y=b} .
$$

\section{Numerical Results}

A square fully clamped plate of unit length with uniform distributed load is studied. The deflection at its mid-point is calculated. This is done with different thickness to side length ratios, starting at a very small ratio and ending at a ratio 0.13 , which is almost the limit of moderately thick plate.

All values for the deflection are normalized so they would be compared to the references. The normalized deflection is given as $\overline{\mathrm{w}}=\frac{\mathrm{wqa}^{4}}{\mathrm{D}}$ where $\mathrm{q}$ is the amplitude of the uniform distributed load.

The plates with the same ratios were modeled using the finite element package ANSYS [21] using two different types of elements. The first type is the Shell181 element which relies on the Reisnner-Mindlin plate theory. The second type is the Block20 3D element which was used to find the 3D solution of the same plates.

The results of the four methods are summarized in table (1).

Figure (4) illustrates the change of the mid-point deflection with the change of the thickness ratio. At small thickness ratios, which can be considered a thin plate, all the solutions converge to the thin plate theory solution which was given In [1].

As the thickness ratio increases the difference increases between the Bergan-Wang approach solutions and the two used finite element solutions which are based on Reissner-Mindlin and the 3D elasticity theory. The relative error related to $3 \mathrm{D}$ solution is given in Table 2 showing that first 
Table 1 The normalized mid-point deflection of a fully clamped plate using different methods (FIT stands for Finite integral transform).

\begin{tabular}{lllllll}
\hline $\mathrm{h} / \mathrm{a}$ & $\left(W_{\mathrm{RM}}\right)$ & $\left(W_{3 \mathrm{D}}\right)$ & $\left(W_{\mathrm{BC}-\mathrm{I}}\right)$ & $\left(W_{\mathrm{BC}-\mathrm{II}}\right)$ & $\begin{array}{l}\mathrm{RE} \\
\left(W_{\mathrm{BC}-\mathrm{I}}\right) \times 10^{3}\end{array}$ & $\begin{array}{c}\mathrm{RE}^{* *} \\
\left(W_{\mathrm{BC}-\mathrm{II}}\right) \times 10^{3}\end{array}$ \\
\hline 0.001 & $\mathrm{x} 10^{3}$ & $\mathrm{x} 10^{3}$ & $\mathrm{x} 10^{3}$ & $\mathrm{x} 0^{3}$ & 0.031 & 0.159 \\
0.01 & 1.265 & - & 1.265 & 1.263 & 0.037 & 0.274 \\
0.02 & 1.268 & - & 1.267 & 1.264 & 0.061 & 0.623 \\
0.03 & 1.275 & - & 1.274 & 1.267 & 0.126 & 1.190 \\
0.04 & 1.288 & - & 1.286 & 1.272 & 0.279 & 1.953 \\
0.05 & 1.305 & 1.318 & 1.301 & 1.280 & 0.550 & 2.896 \\
0.06 & 1.327 & 1.338 & 1.320 & 1.288 & 0.945 & 3.979 \\
0.07 & 1.353 & 1.361 & 1.341 & 1.300 & 1.479 & 5.190 \\
0.08 & 1.385 & 1.391 & 1.364 & 1.313 & 2.199 & 6.569 \\
0.09 & 1.420 & 1.422 & 1.389 & 1.327 & 3.139 & 8.017 \\
0.10 & 1.460 & 1.457 & 1.414 & 1.343 & 4.216 & 9.334 \\
0.11 & 1.504 & 1.492 & 1.441 & 1.364 & 5.308 & 10.427 \\
0.12 & 1.552 & 1.541 & 1.470 & 1.390 & 6.375 & 11.364 \\
0.13 & 1.605 & 1.588 & 1.502 & 1.422 & 7.496 & 12.302 \\
\hline
\end{tabular}

Table 2 The relative error of the normalized mid-point deflection of a fully clamped plate with respect to the 3D solution.

\begin{tabular}{llll}
\hline $\mathrm{h} / \mathrm{a}$ & $\begin{array}{l}* \mathrm{RE} \\
\left(W_{\mathrm{RM}}\right)\end{array}$ & $\begin{array}{l}* * \mathrm{RE} \\
\left(W_{\mathrm{BC}-\mathrm{I}}\right)\end{array}$ & $\begin{array}{l}* * * \mathrm{RE} \\
\left(W_{\mathrm{BC}-\mathrm{II}}\right)\end{array}$ \\
\hline 0.04 & 1.031 & 1.308 & 2.964 \\
0.05 & 0.828 & 1.373 & 3.700 \\
0.06 & 0.555 & 1.495 & 4.512 \\
0.07 & 0.438 & 1.910 & 5.605 \\
0.08 & 0.152 & 2.347 & 6.711 \\
0.09 & -0.211 & 2.935 & 7.823 \\
0.10 & -0.818 & 3.433 & 8.592 \\
\hline
\end{tabular}

$* \operatorname{RE}\left(\mathrm{w}_{\mathrm{RM}}\right)=\frac{\mathrm{w}_{3 \mathrm{D}}-\mathrm{w}_{\mathrm{RM}}}{\mathrm{w}_{3 \mathrm{D}}} \times 100, * * \mathrm{RE}\left(\mathrm{w}_{\mathrm{BC}-\mathrm{I}}\right)=\frac{\mathrm{w}_{3 \mathrm{D}}-\mathrm{w}_{\mathrm{BC}-\mathrm{I}}}{\mathrm{w}_{3 \mathrm{D}}} \times 100$,

$* * * \mathrm{RE}\left(\mathrm{w}_{\mathrm{BC}-\mathrm{II}}\right)=\frac{\mathrm{w}_{3 \mathrm{D}}-\mathrm{w}_{\mathrm{BC}-\mathrm{II}}}{\mathrm{w}_{3 \mathrm{D}}} \times 100$

boundary conditions give better results than those of second boundary conditions.

$\mathrm{w}_{\mathrm{RM}} \quad$ Reissner-Mindlin solution, $\mathrm{w}_{\mathrm{BC}-\mathrm{I}}$ Bergan-Wang solution with first B.C's, $w_{3 D}$ 3D solution, $\mathrm{W}_{\mathrm{BC}-\mathrm{II}}$ Bergan-Wang solution with second B.C's,

$$
\begin{aligned}
& * \mathrm{RE}\left(\mathrm{w}_{\mathrm{BC}-\mathrm{I}}\right)=\frac{\mathrm{w}_{3 \mathrm{D}}-\mathrm{w}_{\mathrm{BCI}}}{\mathrm{w}_{3 \mathrm{D}}} \times 100, \\
& * * \mathrm{RE}\left(\mathrm{w}_{\mathrm{BC}-\mathrm{II}}\right)=\frac{\mathrm{w}_{3 \mathrm{D}}-\mathrm{w}_{\mathrm{BCII}}}{\mathrm{w}_{3 \mathrm{D}}} \times 100
\end{aligned}
$$

To check the convergence of the two methods, the problem of a clamped square thin plate was solved by the two methods described in the previous two sections. The value of the thickness ratio was taken
0.00001 . The number of terms of the series solution was varied and the normalized deflection at the mid-point of the plate was calculated and checked with the classical plate theory solution.

\subsection{Convergence of Galerkin's Method}

It was found that increasing the number of terms in the series makes the solution converges to the classical plate theory solution after taking 10 terms. It was also found that taking an odd number of terms gives a solution more convergent than if any of the two adjacent even numbers is taken as the number of the series terms. This is illustrated in figure (5). 
The solution using this method involves solving a system of linear equations of the order $m^{2}$ for $m=n$.

\subsection{Convergence of Finite Integral Transform method} (FIT)

Using a value of $\mathrm{m}$ and $\mathrm{n}$ equals 300 we could get the value of the mid point normalized deflection of the plate to be 0.001263 . It was found that the convergence of this method is very slow.

The solution using this method involves solving two systems of linear equations each of the order $4 \mathrm{~m}$ for equal values of $m$ and $n$.

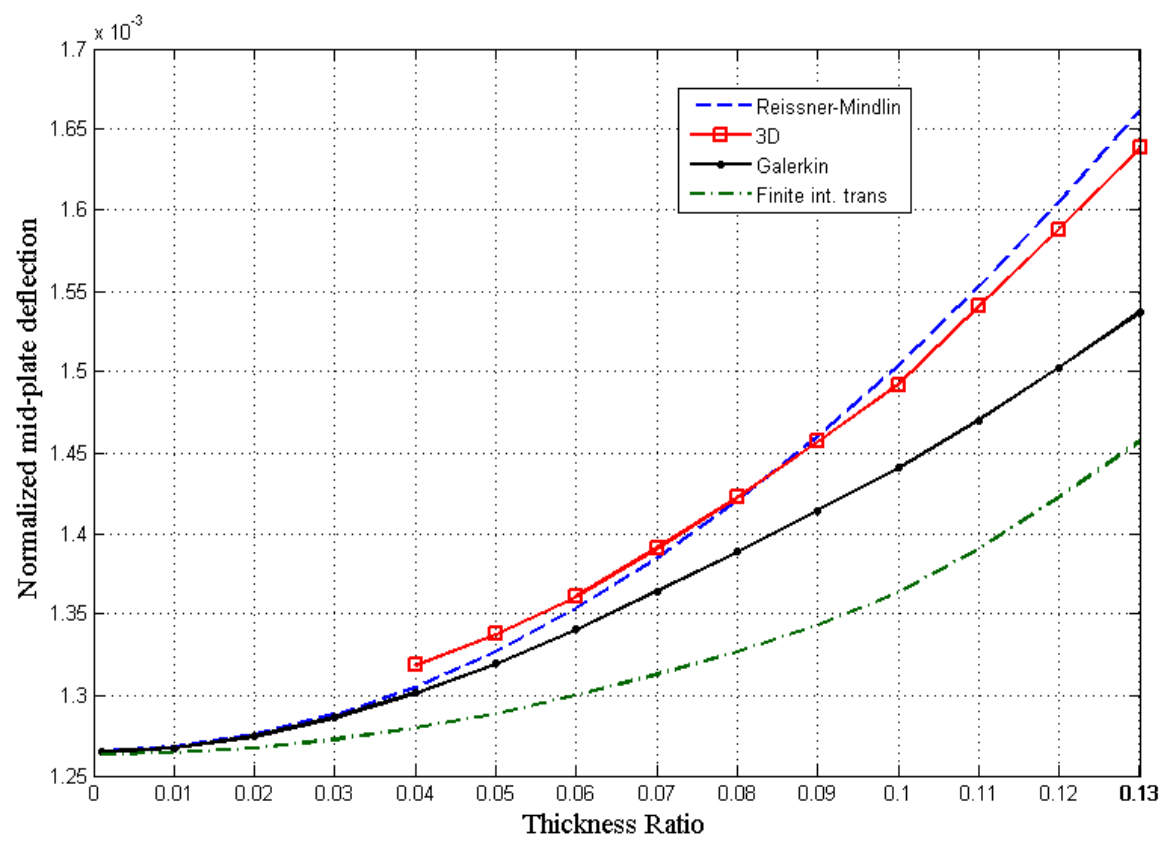

Fig. 4 Comparison between different solutions.

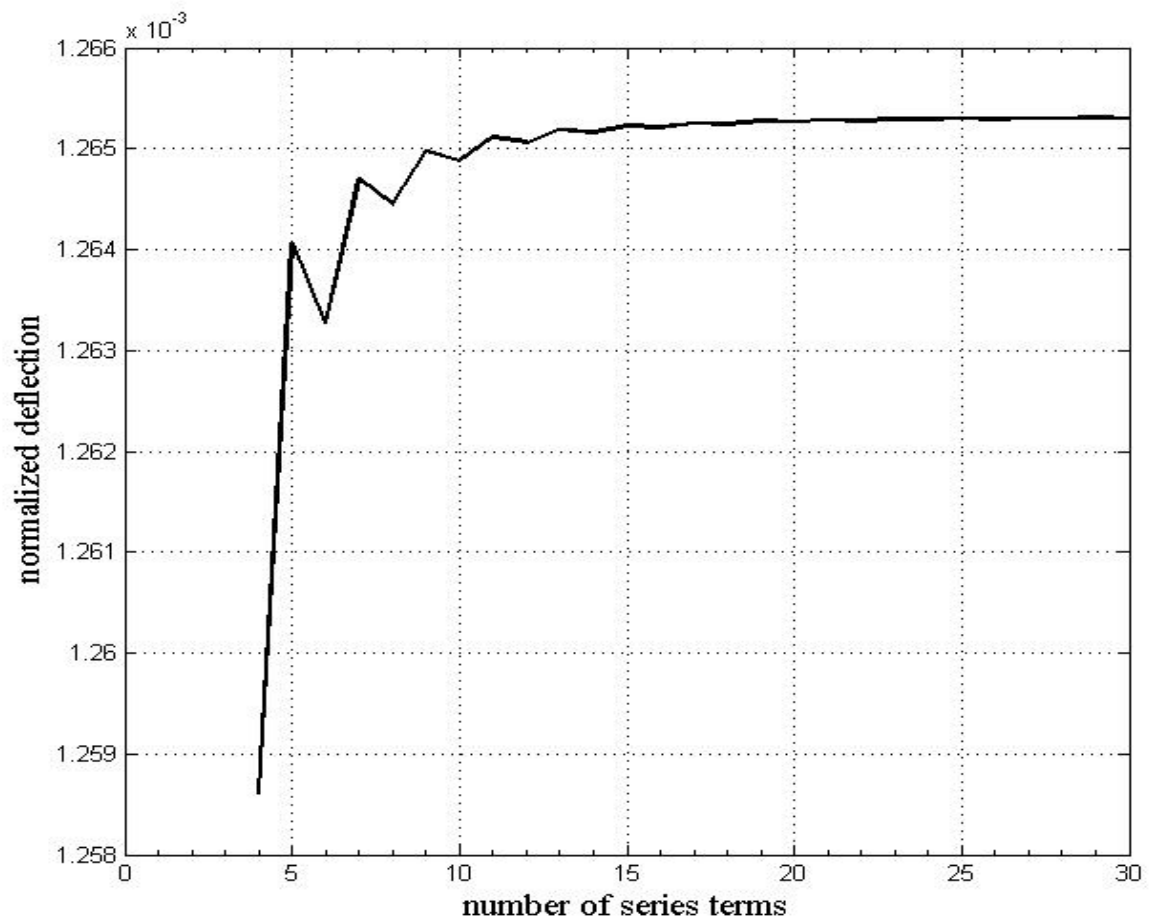

Fig. 5 The convergence of the series solution in Galerkin's method with increasing number of terms. 


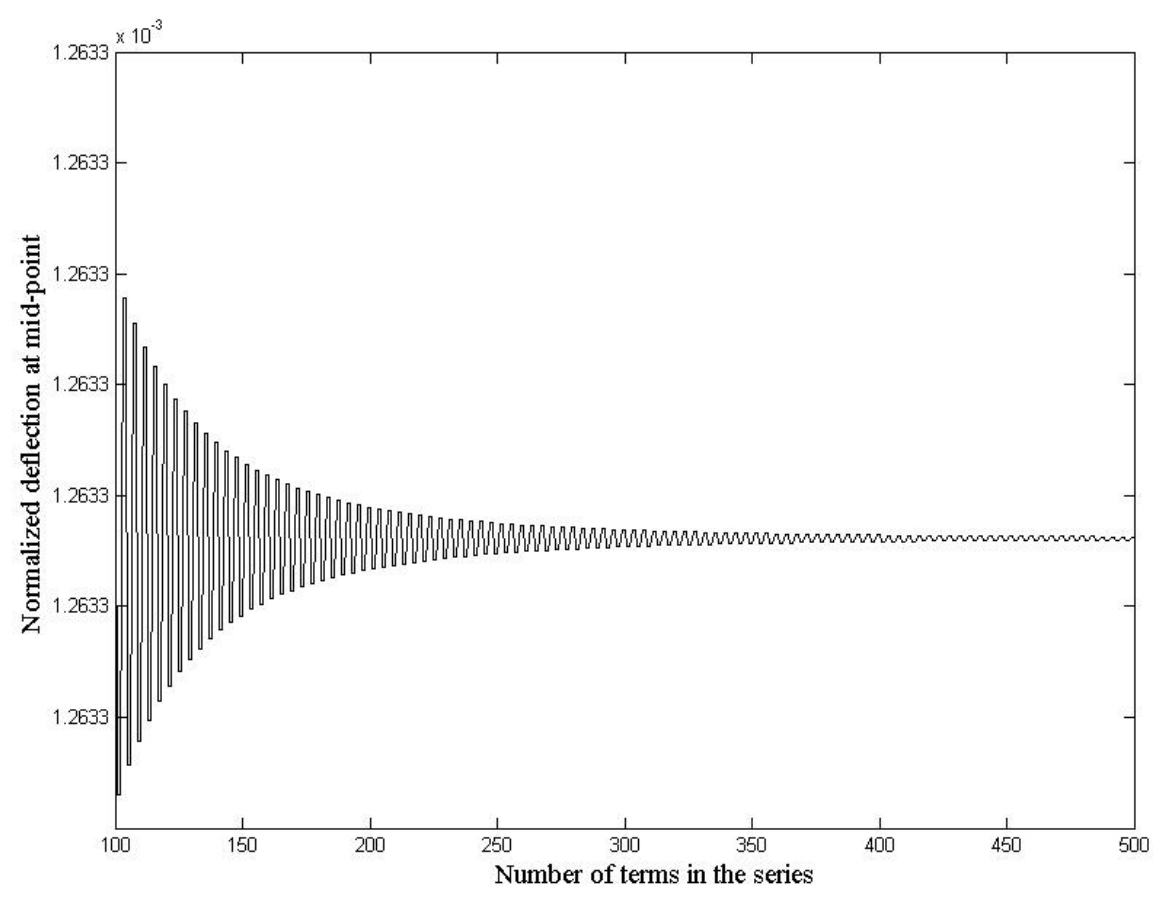

Fig. 6 The convergence of the series solution in FIT method with increasing number of terms

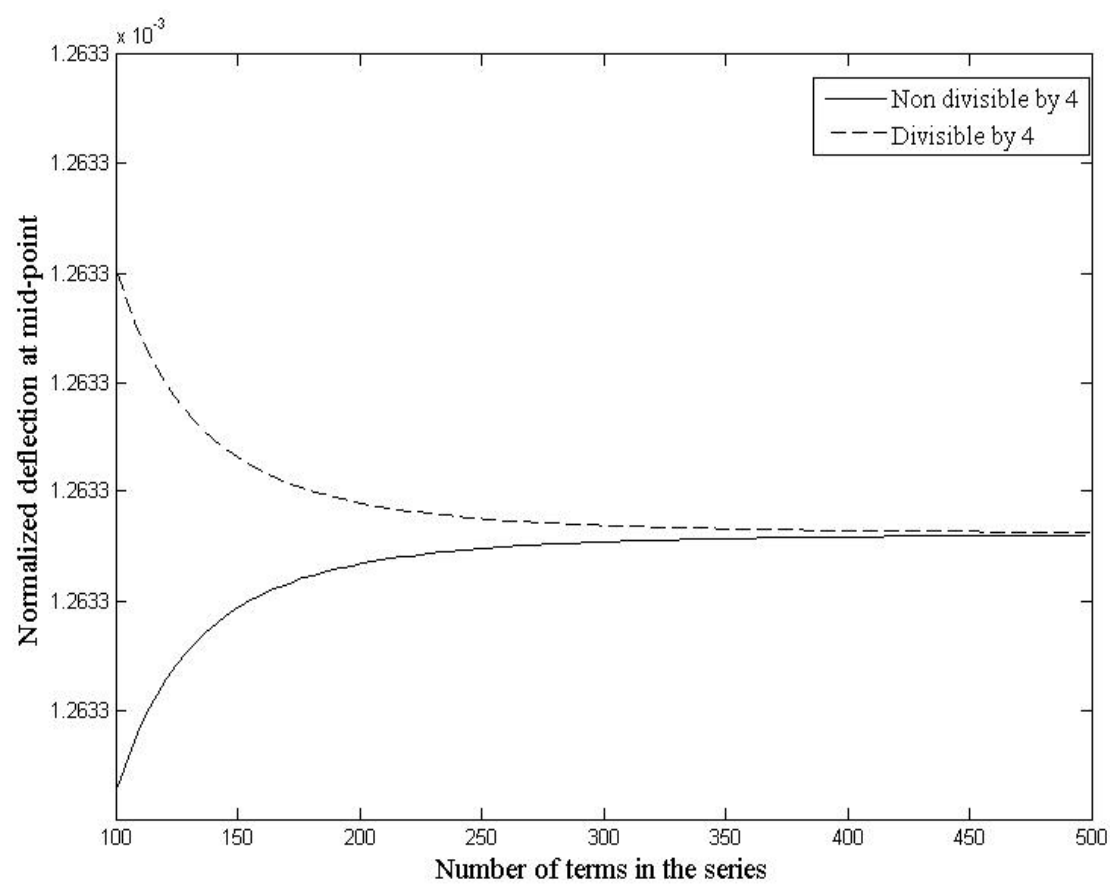

Fig. 7 The asymptotic convergence of FIT solution for even number of terms divisible

Investigating the different values of series terms it was found that:

The odd number of terms gives exactly the same result of the previous even number.
The even number of terms that is divisible by 4 gives a result which is upper asymptotic to the solution while the even number that is not divisible by 4 gives a result which is lower asymptotic to the 
solution. This is illustrated in figures (6) and (7).This makes it important to carefully choose the number of terms in the series.

\section{Conclusions}

The Bergan-Wang approach used in solving for the deflection of plates, proved to have a powerful feature. It can be used for thin, moderately thin and moderately thick plates. The choice of zero values for $w, w_{n}, \theta_{n}$ and $M_{n s}$ as the boundary conditions gave good results for all thickness ratios till the moderately thick plates. Nevertheless, it is still believed that further investigations on the boundary conditions of a fully clamped plate are needed. Further studies are needed to find the effect of the thickness ratio near the abrupt change in thickness, at holes and near the edges. The flexibility of finite element method compared with the series solution can help in this direction. Moreover, the finite element method will clarify the effect of thickness (through the factor $h_{o}{ }^{2}$ ) on the solution at the boundary. A special care is needed in deciding the number of terms used in the series solution. This is due to both the expenses of solving the linear system and convergence.

\section{References}

[1] Timoshenko, S.Woinowski-Krieger. Theory of plates and shells. Singapore: McGraw-Hill.; 1959.

[2] V Meleshko, Bending of an elastic rectangular clamped plate: exact versus engineering solutions, Journal of elasticity. 1997; 48:1-50.

[3] J.N. Reddy, Theory and Analysis of Elastic Plates and Shells. Boca Raton, FL: CRC press; 2007.

[4] D.W Zietlow, D.C Griffin, T.R. Moore, The limitations on applying classical thin plates clamped on the inner boundary, AIP ADVACES.2012; 2: 1-8.

[5] E. Reissner, The effect of transverse shear deformation on the bending of elastic plates, Journal of Applied Mechanics. 1945; 67: 69-77.

[6] R.D.Mindlin, Influence of rotary inertia and shear on flexural vibration of isotropic, elastic plates, Journal of Applied Mechanics.1951; 40:31-38.

[7] G.T Lim, J.N. Reddy, On canonical bending relationships for plates, International Journal of Solids and Structures.2003; 40: 3039-3067.

[8] O.Zienkiewicz, R. Taylor, The finite Element Method for Structural Mechanics.6th Edition, Oxford: Butterworth Heliemann; 2005.

[9] P.G. Bergan, X. Wang, Quadrilateral plate bending elements with shear deformation, Computers and Structures. 1984;19: 25-34.

[10] H. Abdalla, K. Hassan, On the Bergan-Wang approach for moderately thick plates, Communications in Numerical Methods in Engineering. 1988; 4: 51-58.

[11] R.L. Taylor, S. Govindjee, (2004) Solution of clamped rectangular plate problems, Communications in Numerical Methods in Engineering.2004; 20: 757-765.

[12] R.Li, Y. Zhong, B. Tian, Y. Liu, On the finite integral method for exact bending solutions of fully clamped orthotropic rectangular thin plates, Applied Mathematics Letters.2009; 22: 1821-1827.

[13] R.P. Shimpi, Refined plate theory and its variants, AIAA Journal.2002. 40 (1): 137-146.

[14] R.P. Shimpi, H.G. Patel, H.Arya, New first order shear deformation plate theories, Journal of Applied Mechanics.2007; 74: 523-533.

[15] R.J. Craig, (1987) Finite difference solutions of Reissner's plate equations, Journal of engineering mechanics. 1987; 118: 31-48.

[16] B. Haggblad, K.J. Bathe, Specifications of boundary conditions for Reissner/Mindlin plate bending finite elements, International journal for numerical Methods.1990; 30: 981-1011.

[17] B. G. Galerkin, Rods and plates: series in some questions of elastic equilibrium of rods and plates, Vestnik in zhenerov I technikov.1915; 19: 897-908.

[18] S.G. Mikhlin, Variational Methods in Mathematical Physics. Oxford:Pergamon Press; 1964.

[19] I.N. Sneddon, The use of integral transforms. New York: McGraw-Hill; 1972.

[20] I.N. Sneddon, (1975) Application of integral transforms in the theory of elasticity. New York: McGraw-Hill; 1975.

[21] Y.Nakasone, S. Yoshimoto, T.A., Stolarski, Engineering Analysis with ANSYS Software. Oxford: Elsevier Butterworth-Heinemann Linacre House, Jordan Hill; 2006. 